\section{Standard Theory of Particle Interactions}

\section{Maiani, Rome}

(Università "La Sapienza")

Particle physics is a relatively young subject. The atomic nucleus was discovered only at the beginning of this century and the neutron identified in the thirties. Furthermore, only since the second world war has the richness of the subnuclear particle spectrum been revealed as accelerators of steadily increasing energy have become available, and the first relativistic quantum field theory, Quantum Electrodynamics or QED for short, been fully developed.

Pre-war physics had already identified the interactions which dominate particle physics: the strong nuclear interaction, the electromagnetic interaction and the weak interaction, responsible for nuclei and $\beta$-particle decays. This allowed a first classification of particles into two broad categories. The hadrons, particles such as the proton, the neutron, the $\pi$ meson etc., which respond to the strong interaction (as well as to the others) and the leptons, particles which are subject to the electromagnetic and weak forces only. The lepton category is known today to include the electron, the muon and the recently discovered $\tau$-lepton, together with three corresponding kinds of neutrinos (being electrically neutral, neutrinos interact only through the weak force).

In a relativistic quantum field theory, forces are themselves transmitted by particles, so there should be a third category, that of the force carriers. Of the latter, only the photon, the go-between of the electromagnetic interaction, was known to pre-war physics. The strong interaction was supposed to arise from the exchange of the Yukawa $\pi$-meson and the weak force was suspected to be transmitted by a hypothetical heavy particle, later called the intermediate weak boson. The present theory of particle interactions, the Standard Theory, makes a much simpler and definite statement: all forces are transmitted by spin one (photon like) particles, universally coupled to the matter fields through a set of conserved, or only softly broken, charges.

Great progress has also been made in our understanding of the hadrons. Spec- troscopic evidence has made it clear that hadrons are composite states of spin $1 / 2$ objects, named quarks by their inventor, M. Gell-Mann. Quarks are the really elementary constituents of hadronic matter, or at least they appear to be so up to the highest energies so far explored. In close analogy with the three lepton families $\left(v_{e}, e\right),\left(v_{\mu}, \mu\right)$ and $\left(v_{\tau}, \tau\right)$, the Standard Theory requires the existence of three quark families, denoted by $(u, d),(c, s)$ and $(t, b)$. The $u, d$ and $s$ (up, down and strange) quarks account for the normal nuclear matter and for the strange particles; the charmed, c, and beauty, $b$, quarks are the constituents of the charmed and beauty particles discovered in more recent years. Firm experimental evidence for particles made of $\mathrm{t}$-quarks is still lacking.

\section{Symmetry}

A crucial role in the standard theory is played by the notion of internal, continuous symmetry. The concept was first introduced by W. Heisenberg in the thirties, to account for the close similarity of the nuclear interactions of protons and neutrons. The isospin symmetry and its further extensions have been very useful in classifying the levels and the selection rules of the hadronic spectrum.

The further step, which leads directly to the present theory of the weak and electromagnetic interactions, is to use the symmetry to determine the interaction, rather than simply restrict it. The way this comes about is analogous to the way the minimal interaction is introduced in OED. There, the substitution:

$$
\mathrm{p}_{\mu} \rightarrow \mathrm{p}_{\mu}-e Q A_{\mu}
$$

where $Q$ is the electric charge in units of the proton charge $e$, and $A_{\mu}$ is the vector potential, guarantees the symmetry under local gauge transformations. At the same time, it gives rise to a completely prescribed interaction. Similarly, if we denote by $T^{i}$ the generators of some internal symmetry group $G$, the substitution:

$$
\mathrm{p}_{\mu} \rightarrow \mathrm{p}_{\mu}-g \sum_{i} T^{i} A_{\mu}^{i}
$$

gives rise to a unique interaction of the matter with the vector fields $A_{\mu}^{i}$, which is symmetric under the local symmetry transformations of $G$, provided we subject the $A_{\mu}^{i}$ to an appropriate gauge transformation (Yang, Mills 1954).

It takes only a little more mathematics, and a good dose of hindsight, to show that the substitution eq. (2) is able to generate interactions which include the familiar electrodynamics $(T=Q)$ as well as the universal $\beta$-decay interaction, assumed to be mediated by charged vector particles. Definitely less simple to understand is how the symmetry is broken, so as to produce at the same time massive weak vector bosons and a massless photon. As in quantum electrodynamics, exact gauge invariance implies that the vector potential describes a massless particle. Gauge invariance has to be broken if weak interactions are mediated by intermediate bosons as they are observed to be. How then do we make all this into a theory which is mathematically consistent (read: renormalizable) as QED is by itself? This can be obtained if scalar fields are present whose vacuum expectation value breaks $G$, leaving the gauge transformations of QED exact (Higgs mechanism).

When all is said and done ${ }^{1}$ ), one obtains the following picture: The minimal group needed for realistic weak and e.m. interactions is:

$$
G=\mathrm{SU}(2) \times \mathrm{U}(1)
$$

It requires four vector particles (one for each group generator) and two indepen-

Table 1 - Values of $\sin ^{2} \theta$ determined in different experiments, see the reviews in ref. (2) for more details and for reference to the experimental papers. The last column gives the radiative correction which has been applied to the value of

\begin{tabular}{|c|c|c|}
\hline Experiment & $\mathrm{S}^{2}$ & RAD. CORR. \\
\hline $\begin{array}{l}\text { ATOMIC P.V. (Caesium) } \\
\text { (Paris) }\end{array}$ & $0.230 \pm 0.030$ & +0.009 \\
\hline $\begin{array}{l}\text { e-D ASYMMETRY } \\
\text { (SLAC) }\end{array}$ & $0.218 \pm 0.020$ & -0.010 \\
\hline $\begin{array}{l}\mathrm{v}_{\mu}-\mathrm{e} \\
(\mathrm{BNL}+\mathrm{CHARM})\end{array}$ & $0.212 \pm 0.023$ & SMALL \\
\hline $\begin{array}{l}v_{\mu}-P(E L A S T I C \\
\text { SCATT.) (BNL) }\end{array}$ & $0.220 \pm 0.031$ & SMALL \\
\hline $\begin{array}{l}v_{\mu}-N \text { (DEEP INEL. } \\
\text { SCATT.) } \\
\text { (CDHSW + CHARM } \\
+ \text { CCFRR + FFM) }\end{array}$ & $\begin{array}{l}0.233 \pm 0.003 \\
\pm 0.006\end{array}$ & -0.011 \\
\hline $\begin{array}{l}\mathrm{M}_{\mathrm{W}, \mathrm{Z}} \\
\text { (UA1+UA2) }\end{array}$ & $\begin{array}{l}0.227 \pm 0.003 \\
\pm 0.008\end{array}$ & +0.016 \\
\hline $\begin{array}{l}1-M_{W}^{2} / M_{2}^{2} \\
\text { (UA1+UA2) }\end{array}$ & $0.218 \pm 0.022$ & - \\
\hline
\end{tabular}
$\sin ^{2} \theta$, to make it agree with the definition in eq. (8) of text. 
dent couplings (one for each commuting sector). The lowering and raising generators of $\mathrm{SU}(2)$ transform, e.g. $\mathrm{v}_{\mathrm{e}}$ into $\mathrm{e}$, or u-quarks into d-quarks (and viceversa). The corresponding vector bosons, $W^{ \pm}$, are therefore electrically charged, and mediate the usual $\beta$ decays. Of the other two, one vector field is the photon, but there remains a fourth neutral boson, denoted by $Z$, which couples universally to a combination of the e.m. current and of the current associated with the third generator of $\mathrm{SU}(2)$ :

$L_{Z}=(g / \cos \theta) Z^{\mu}\left(J_{\mu}{ }^{(3)}-\sin ^{2} \theta j_{\mu}^{\text {e.m. }}\right)$ where $g$ denotes the coupling constant associated with $\mathrm{SU}(2)$ and $\theta$ a new parameter, related to $\mathrm{g}$ and to the electric charge:

$$
\sin \theta=(e / g)
$$

The $\mathrm{W}^{ \pm}$and $\mathrm{Z}$ masses are predicted to be:

$$
\begin{gathered}
M_{\mathrm{w}}=(\pi \alpha / \sqrt{ } 2 G)^{1 / 2} / \sin \theta \\
M_{\mathrm{z}}=M_{\mathrm{w}} / \cos \theta
\end{gathered}
$$

with $G$ and $\alpha$ the Fermi and fine-structure constants.

The existence of new processes, mediated by $Z$, and the mass relations (6) and (7) are the most outstanding predictions of the Standard Electroweak Theory. Z-mediated processes have been carefully scrutinized and can be classified as follows.

i) Parity violation in atomic levels (due to the interference of Z-exchange with the Coulomb potential);

ii) asymmetries in the scattering of longitudinally polarized electrons off a nucleus, e.g. deuterium, again due to $\gamma$-Z interference;

iii) neutrino, Z-mediated, scattering off atomic electrons or nuclei.

The observation of any of the above processes leads to a value of the new parameter, $\sin ^{2} \theta$, and the consistency of the various values gives, of course, a significant test of the electroweak theory itself. The results accumulated over the last decade are summarized in Table 1. Notice that processes i) to iii) involve energies from the atomic level up to the highest energies of the available $v$ beams (corresponding to a centre of mass energy of the order of $20 \mathrm{GeV}$ ) a span of more than nine orders of magnitude.

The discovery of $\mathrm{W}^{+}$and $\mathrm{Z}$ is a great success. To make this more quantitative we can use the measured masses to obtain two other determinations of $\sin ^{2} \theta$, see eqs.(6) and (7). The most recent values are again given in Table 1 , and seem to agree quite remarkably with the previous determinations.

As in any renormalizable theory, the various processes are affected by finite and calculable higher order corrections. Table 1 gives the correction to the lowest order value of $\sin ^{2} \theta$ in each process, needed to make it agree with the conventional, but convenient, definition:

$$
\sin ^{2} \theta \equiv 1-M_{\mathrm{W}}^{2} / \mathrm{M}_{\mathrm{Z}}^{2}
$$

The correction is still quite comparable with the experimental errors, but there is a systematic tendency for it to make the different values of $\sin ^{2} \theta$ agree better. More precise determinations of $Z$ and $W$ mass, with SLC and LEP, and of the $v_{\mu}-\mathrm{e}$ cross-section (CHARM II) should improve significantly on this point.

\section{Colour}

Perhaps the most spectacular aspect of the Standard Theory is that the strong interactions which bind quarks inside the hadrons are also described by a gauge interaction, similarly to the electroweak sector.

This time, the vector fields (gluons) couple to a hidden symmetry of the quarks, conventionally dubbed colour. We do not see colour in the hadronic spectrum, since hadrons are all colour singlets, but we can detect its presence in many ways (for instance, the quark wave-function in the proton is symmetric in all other quantum numbers; it is colour which restores the Pauli principle).

Colour is an exact symmetry, and gluons are massless; yet we do not observe gluons to come out of the hadrons in high-energy collisions. This peculiar phenomenon (colour confinement) is believed to be related to the behaviour of the interaction strength with the distance, which can be studied with renormalization-group techniques. In a Yang-Mills theory, it is found that the effective coupling between two coloured objects decreases when the distance is reduced (asymptotic freedom) and, conversely, it increases for large distances. Thus, if we try to separate e.g. a quark from a gluon, the interaction energy increases up to a point where quark pairs are created from the vacuum which neutralize the colour of the gluon and make it appear in the form of a jet of colour-neutral particles.

Asymptotic freedom is the key concept, which has made it possible to test quantitatively the theory of quarks and gluons (Quantum Chromodynamics, QCD for short) although we have never observed either a quark or a gluon. In processes involving large momentum transfer, i.e. short distances, we can use perturbation theory to obtain meaningful predictions. One such is illustrated in Fig. 1, where the transverse momentum distribution of $\mathrm{W}$ bosons produced in $\mathrm{p}-\overline{\mathrm{p}}$ collisions is reported:

$$
\mathrm{p}+\overline{\mathrm{p}} \rightarrow \mathrm{W}+\text { hadrons }
$$

The long tail, at $p_{T}^{W} \gtrsim 10 \mathrm{GeV}$, arises in the theory mainly from the emission of a single hard gluon, which is seen as an energetic jet of normal hadrons in the apparatus. The QCD prediction agrees with the experimental cross-section also in the absolute normalization. This is interesting because to produce the $\mathrm{W}$, a colour singlet, a quark in the proton must find an antiquark in the antiproton, precisely of the opposite colour, which gives a factor three reduction in probability over the case had quarks no colour.

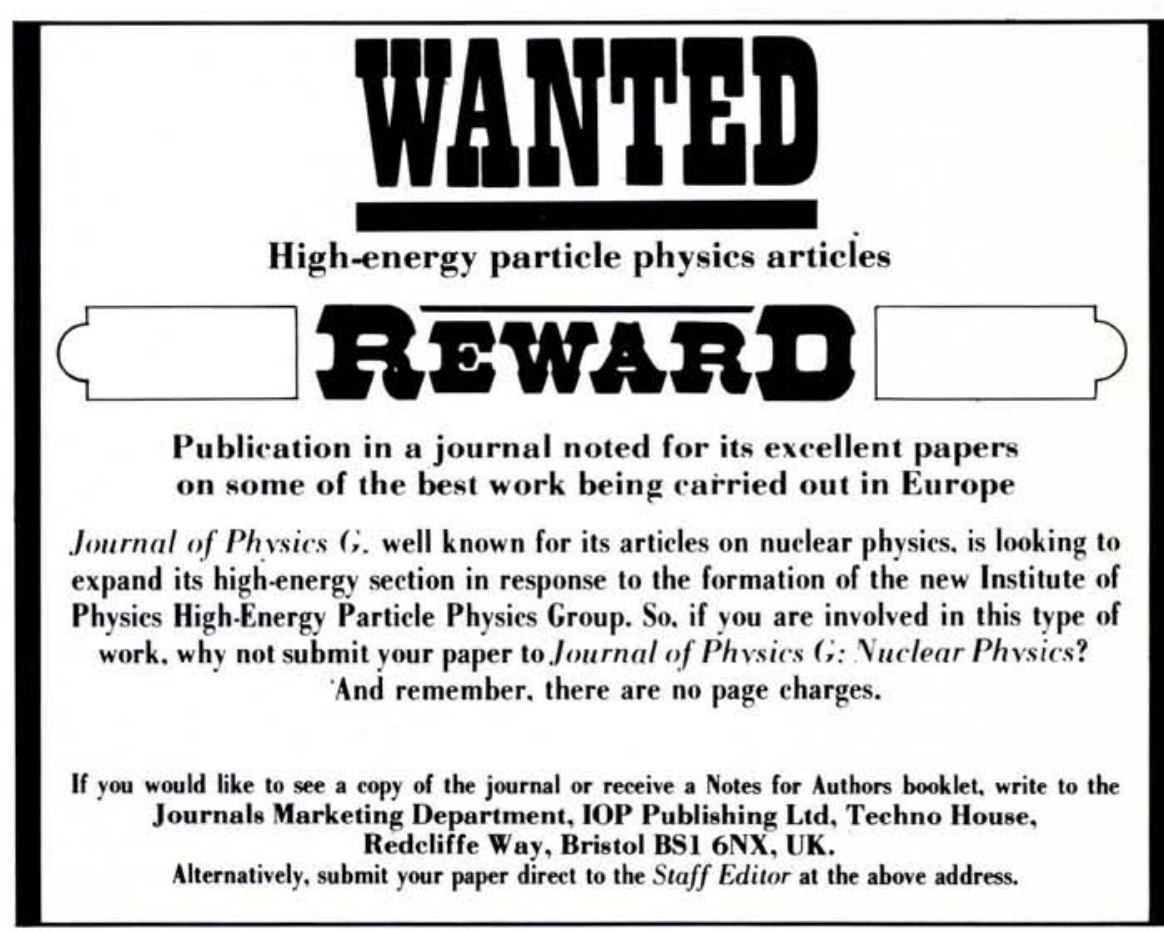


The data definitely support coloured quarks.

\section{Open Problems}

The fact that all particle interactions are described with the same type of Yang-Mills theory leaves ample ground to suspect that a further embedding of the Standard Theory into a more unified structure is needed. The peculiar absence of gravity in the present scheme is another valid reason.

It must be stressed, however, that there still remain many areas, within the theory itself, which need further theoretical and experimental investigation.

A definitive consequence of the way the electroweak symmetry is broken is the existence of a neutral spinless particle, the Higgs boson, which has escaped detection so far. The reason why it is so difficult to observe is that it couples to the particle mass, i.e. it is only feebly coupled to the light matter in the available beams. The next generation of machines should produce abundantly various types of heavy particles, $\mathrm{W}, \mathrm{Z}$ and perhaps t-quarks, and make the observation of the Higgs boson possible. Until this particle has been definitely observed, we cannot claim to understand where $W$ and $Z$ get their mass from.

Short distance processes, where perturbative $Q C D$ can be applied, are only the tip of an iceberg made by the wealth of data from non-perturbative processes (hadronic spectra, cross-sections etc.). The present, most promising approach to non perturbative $\mathrm{QCD}$ is computer simulation of the theory on a discrete lattice. Aside from practical results, it is hoped that the large efforts being made in this direction will give valid clues for a more direct and analytic approach.

\section{REFERENCES}

1. The Standard Theory is described in many textbooks, see e.g. Lee T.D., Particle Physics and Introduction to Field Theory (Harwood Academic Publ. Chur, London, New York) 1981.

2. Altarelli G., Proceedings of the 23rd International Conference on High Energy Physics, Berkeley July 16-23, 1986; Marciano W. op. cit.

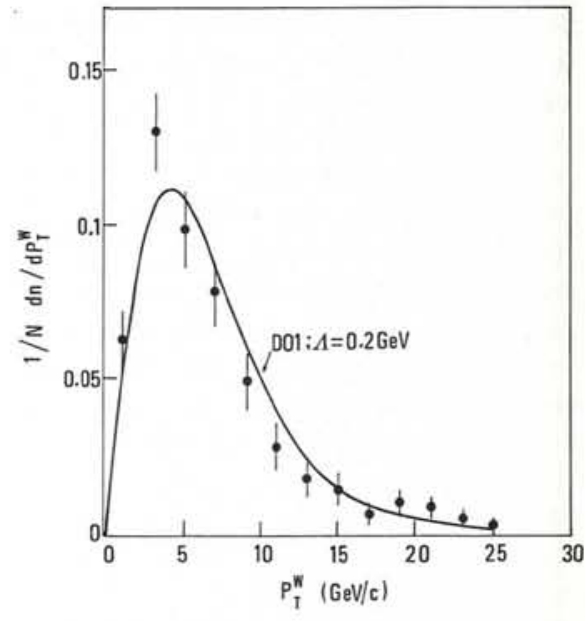

Fig. 1 - Transverse momentum distribution of the $W$ produced in $p-\bar{p}$ collisions at the CERN collider; $P_{T}^{W}$ is the $W$-momentum transverse to the beam. The experimental points are from the UA1 collaboration. The continuous line is the $Q C D$ prediction, normalized to the total number of events, including the experimental resolution and cuts. The theoretical prediction is computed with the structure functions of Duke and Owens, DO1, see ref. 2.

\section{The Evolution of Particle Accelerators}

\section{Bryan W. Montague, Geneva}

(CERN)

Over the past forty years or so, particle accelerators have undergone tremendous developments, both in energy and in design subtlety. Each energy region explored has revealed new features of the structure of matter, but has also raised further questions that required higher energies for their elucidation.

Progress in this field has come about both by major conceptual advances and by a steady evolution in theoretical understanding and applied technology. The phase-stability principle and the invention of alternating-gradient focussing had a major and lasting impact, and the concept of colliding beams has become indispensable for elementary-particle physics at the higher energies.

It had been recognised early on that, in collisions between particles of equal energy moving in opposite directions, the combined energy of both particles is available for the reaction. This contrasts with particles incident on a fixed target, where much of the energy goes into moving the centre of mass. However, to exploit the kinematical advantage of colliding beams requires high beam densities in order to obtain adequate interaction rates or, in other words, sufficient luminosity. By the 1960's the understanding of accelerator beam dynamics together with advances in the technology, made it possible to build useful collidingbeam facilities. By this time also their introduction was becoming really necessary because of the size that accelerators had reached.

In the past fifteen years, collidingbeam physics has been pursued in storage rings of roughly circular shape. These machines fall into two main categories, electron-positron colliders and hadron colliders, the latter producing proton-proton (p-p) or proton-antiproton $(p-\bar{p})$ collisions. Both types obtain sufficiently high beam densities by the accumulation of many injected bunches of particles. Production of anti-proton beams of sufficient density became possible thanks to a major innovation, namely stochastic cooling. This relies on progressively applying correction signals derived from average measurements over a cross-section, to a circulating beam in which continuous mixing of the particles occurs. The net result is a reduction in the relativespread as the beam cools.

Future Needs of High-Energy Physics

Accelerators in the future will be colliding-beam machines either for electrons $\left(\mathrm{e}^{+}-\mathrm{e}^{-}\right)$or for hadrons. The difference between these is significant in several respects. First the point-like nature of electrons yields very "clean" physics with relatively little background, and the full energy of the collision is available for producing other particles and states. In contrast, the quark structure of hadrons leads to much of the collision energy appearing in the form of rather uninteresting "soft" interactions, making the "hard" quark-quark collisions less numerous and more difficult to investigate. Consequently, electron colliders are desirable for detailed precision measurements of high-energy processes, whereas hadron colliders have certain advantages for making new discoveries.

For future electron colliders we shall have to abandon the circular storagering shape because of energy loss through synchrotron radiation, which increases with the fourth power of the energy. The Large Electron Positron (LEP) machine under construction at CERN, with a circumference of $27 \mathrm{~km}$ 